\title{
Salvage versus adjuvant radiotherapy after radical prostatectomy: argument for adjuvant radiotherapy
}

\author{
Joseph L. Chin, MD, FRCSC
}

$\mathrm{T}$ he optimal management for patients at high risk of local recurrence after radical prostatectomy (i.e., those with substantial positive surgical resection margins, with extracapsular extension, with seminal vesicle involvement or a combination thereof) is still a contentious issue. One should emphasize that the controversy concerns those patients with "substantial" or "significant" positive resection margins at multiple sites as opposed to the unifocal positive at the apex. In this latter situation, there is general agreement that observation rather than early adjuvant radiotherapy (ART) is preferable. The main debate is between early ART and salvage radiotherapy (SRT) with biochemical or clinical relapse in those at higher risk for disease recurrence.

Opponents of ART would cite the following arguments:

- only a certain percentage of "high-risk" patients have local failure and SRT is probably just as effective. Thus rather than unnecessarily subjecting every patient to treatment, one can wait until cancer recurrence is confirmed before proceeding with treatment;

- systemic failures, which may occur with or without local recurrence, cannot be addressed by ART;

- toxicity from ART may outweigh potential benefits. Up until recently, critics of ART have also cited the lack of overall survival benefit for ART.

These concerns can be addressed by consulting the considerable amount of evidence in the literature.

\section{Evidence from randomized controlled trials}

Although there have been no randomized trials specifically comparing ART and SRT, there is level I evidence from 2 published randomized series comparing ART with no ART (various delayed therapies including SRT were employed in the latter group). The 2 studies were the European Organization for Research and Treatment of Cancer (EORTC) study $22911^{1}$ and the Southwest Oncology Group (SWOG) study 8794 (including NCIC [National Cancer Institute of Canada] PR-2), ${ }^{2}$ with 1005 and 410 patients, respectively. Both showed progression-free survival advantage with early radiotherapy: $74.8 \%$ versus $52.6 \%(p<0.001)$ for the EORTC study and $67 \%$ versus $48 \%(p<0.001)$ for the SWOG/NCIC study, in favour of ART, with a hazard ratio (HR) of 0.52 . For SWOG 8794/PR-2, metastatic-free survival was $84 \%$ versus $69 \%$ at 5 years, and $68 \%$ versus $49 \%$ at 10 years with an HR of 0.62 (confidence interval $0.46-0.82, p=0.001$ ).

The overall results of randomized series, including a series with 385 participants from Germany published only in abstract form thus far, ${ }^{3}$ indicate a $20 \%-50 \%$ reduction in risk of prostate-specific antigen (PSA) relapse and disease recurrence, with improved biochemical progression-free survival and local control. On meta-analysis of 1743 patients from the 3 randomized trials, ART resulted in improved biochemical progression-free survival $(\mathrm{HR}=0.47, p<0.001)$ and deferred requirement for adjuvant therapies (radiation and androgen ablation) with their associated adverse effects. ${ }^{4}$

The biggest criticism directed at ART is that, up until recently, there had been no benefit in overall survival, likely because of the low number of deaths in either group. However, an updated analysis of SWOG 8794, presented at the 2008 American Urological Association Annual Meeting, shows increased metastatic-free survival $(p=0.021)$ and increased overall survival (median $15.2 \mathrm{yr}$ compared with $13.5 \mathrm{yr}, p=0.031$ ), in addition to increased biochemical control $(p<0.001)$ for the ART group, indicating unequivocal benefit for ART with level I evidence. ${ }^{5}$

Traditionally, patients with seminal vesicle invasion (SVI) on their radical prostatectomy specimens are considered very high risk for systemic disease and thus are viewed as less than ideal candidates for ART. However, with a median follow-up of 12.2 years, the SWOG 8794/PR-2 trial showed that those with SVI who received ART compared with those who were initially observed had an improved 10 years free from biochemical failure (FFBF) survival from $12 \%$ to $36 \%$

The purpose of the Point / Counterpoint section is to encourage vigorous and informed discussion on controversial issues in urology through the presentation of diverse opinions. We aim for a dispassionate discussion of controversies, recognizing that strong passions may exist in support of some positions. 
$(p=0.001)$ and 10 -year overall survival from $51 \%$ to $71 \%{ }^{6}$ Thus, even with SVI, some patients appear to benefit from adjuvant local radiotherapy.

\section{Evidence from nonrandomized series}

At a lower level of evidence, there have been many nonrandomized series from various countries comparing ART versus SRT or ART versus surveillance with delayed treatment. The results consistently showed significant reduction in long-term biochemical progression with ART. The 5-year biochemical disease-free (bNED) rates range from $61.3 \%$ to $90.9 \%$ for the ART groups and $31 \%$ to $66.4 \%$ for the delayed therapy groups. Three series have reported superior 10-year bNED rates for the ART group compared with the SRT group: Teh and colleagues ${ }^{7}$ with $90.9 \%$ versus $54.5 \%(p=0.001)$, Caraffini and colleagues ${ }^{8}$ with $38 \%$ versus $28 \%(p<0.01)$ and Pacholke and colleagues ${ }^{9}$ with $66 \%$ versus $22 \%(p<0.001)$.

Some groups attempted matched-control analyses of ART versus SRT. ${ }^{10,11}$ Trabulsi and colleagues ${ }^{10}$ used a multiinstitutional database with 211 and 238 patients receiving ART and SRT, respectively. The 5-year FFBF from the time of completion radiotherapy was $73 \%$ for ART versus $50 \%$ for SRT $(H R=2.3, p<0.001)$. Similarly, Wadasaki and colleagues ${ }^{11}$ reported, on a smaller cohort, a 3 -year biochemical control rate of $87 \%$ for ART versus $61 \%$ for SRT patients. For those with a preradiotherapy serum PSA level less than $0.5 \mu \mathrm{g} / \mathrm{L}$, the biochemical control rate was $92 \%$ with ART.

Several case series provide low-level evidence on the beneficial effects of initial observation and delayed SRT after radical prostatectomy. ${ }^{12-16}$ Loeb and colleagues ${ }^{12}$ reported impressive long-term undetectable PSA rates on patients with positive surgical margins and/or extracapsular extension and even those with SVI ( $91 \%$ and $75 \%$, respectively). This would suggest SRT is preferable. However, the patients were not randomly assigned to treatment groups and the results may well be spuriously favourable since those for whom treatment failed early or for whom treatment was likely to fail systemically would have been excluded from the SRT group. Long-term success with SRT is less than $50 \%$ in a multi-institutional cohort report on 1540 patients, ${ }^{13}$ even with early intervention.

\section{Conditions for optimal results from radiotherapy after surgery}

One needs to consider factors that would optimize results from radiotherapy after radical prostatectomy. Pisansky and colleagues $^{17}$ reported the effect of serum PSA levels at the time of SRT on the outcome. The bNED rates for those with a preradiotherapy serum PSA level of less than $0.2 \mu \mathrm{g} / \mathrm{L}$, $0.2-2.0 \mu \mathrm{g} / \mathrm{L}$ and greater than $2.0 \mu \mathrm{g} / \mathrm{L}$ were, respectively, $77 \%, 59 \%$ and $10 \%(p<0.001)$. Stephenson and colleagues ${ }^{13}$ performed a multivariate analysis and reported that preradiotherapy serum PSA levels were a predictor of progression with an HR of $2.3(p<0.001)$ for serum PSA levels less than $2.0 \mu \mathrm{g} / \mathrm{L}$ or greater than $2.0 \mu \mathrm{g} / \mathrm{L}$. A Japanese study reported a 3-year biochemical control rate of $93 \%$ and $29 \%$ for those with preradiotherapy serum PSA levels of $0.5 \mu \mathrm{g} / \mathrm{L}$ or less, and greater than $0.5 \mu \mathrm{g} / \mathrm{L}$, respectively. ${ }^{11}$ Thus, ideally, SRT should be administered when the serum PSA level is less than $0.5 \mu \mathrm{g} / \mathrm{L}$, or as close to the adjuvant situation as possible.

Proponents of SRT would cite the utility of serum PSA levels in guiding timing for SRT to achieve optimal results. The measurement of serum PSA levels alone, however, is less than ideal for detecting the earliest evidence of cancer recurrence. Functional imaging techniques may prove to be useful, for example, positron emission tomography or computed tomography with $18 \mathrm{~F}$ choline and $11 \mathrm{C}$-acetate, magnetic resonance imaging and ProstaScint scanning. ${ }^{18,19}$ However, these modalities are at various stages of development and, until the positive and negative predictive values for local recurrence after radical prostatectomy are improved, delaying therapy for many patients may mean denying them of potential cure.

\section{Safety and toxicity considerations}

An argument against ART is the potential for treatmentrelated toxicity, especially if some patients may not benefit from the treatment. In the adjuvant setting only, a lower dose is required (60-65 Gy), as opposed to the salvage setting, in which 66-70 Gy would be required. ${ }^{20}$ In terms of concerns about toxicity with radiotherapy in the early postoperative setting, Van Cangh and colleagues ${ }^{21}$ randomly assigned 48 patients to early ART (60 Gy) versus 52 patients to "watchful waiting." At 24 months, there was no difference in complete urinary continence ( $77 \%$ v. $83 \%)$. Feng and colleagues ${ }^{22}$ analyzed results on post-radical prostatectomy patients who received either ART (19\%) or SRT (81\%). At 5 years, $10 \%$ had grade 2 and $1 \%$ had grade 3 late genitourinary toxicity. ${ }^{22}$ There were no significant factors on multivariate analysis for gastrointestinal toxicity.

Health-related quality of life was compared prospectively by Pinkawa and colleagues ${ }^{23}$ after ART and SRT in 101 patients. The authors noted that mild bowel symptoms did require longer to recover than urinary symptoms, although 1 year after radiotherapy there were only minor 
health-related quality of life changes. Likewise, data from the SWOG 8794/PR-2 trial indicated only short-term toxicity with early adjuvant radiotherapy, which was well tolerated. Grade 1 or 2 gastrointestinal toxicity at 6 weeks occurred in $59 \%$ of patients in the radiotherapy group versus $7 \%$ in the observation group $(p<0.001)$, but at 5 years, there was no difference (11\% v. $19 \%, p=0.16)$. Similarly, genitourinary toxicity occurring at 6 weeks was $37 \%$ versus $18 \%(p=0.004)$ and at 5 years, there was no difference $(23 \%$ v. $18 \%, p=0.55) .^{2}$

\section{Additional beneficial effects of ART}

The SWOG 8794/PR-2 study also provides level I evidence that the onset of hormonal therapy use was delayed in patients who had undergone ART (9\%) compared with the $20 \%$ in the observation arm at 5 years $(\mathrm{HR}=0.44, p<$ 0.001). ${ }^{2}$ Thus, for the ART group, there are positive quality of life and economic implications as well as possible deference of onset of hormone resistance.

\section{Other cancer sites}

Lastly, one can examine the evidence from other paradigms. In an EORTC study, breast cancer patients undergoing lumpectomy were randomly assigned to ART versus no radiotherapy, and there was a $47 \%$ reduction of local recurrence at 10 years in the ART arm. Similar reports have been published for colon cancer ${ }^{24}$ and head and neck cancers. ${ }^{25}$ Should prostate cancer be any different?

\section{Summary}

The benefits of early radiotherapy in other paradigms have been established. There is level I evidence that ART has acceptable, well-tolerated transient toxicity, which dissipates with time. The best conditions for optimal results from radiotherapy occur when the serum PSA level is very low or undetectable, that is, in the true adjuvant setting. There is substantial evidence from nonrandomized series, almost unanimously in favour of ART for biochemical recurrence rates. There is robust level I evidence from well-executed randomized clinical trials reporting superior biochemical relapse-free and progression-free survival with early ART for patients at high risk for tumour recurrence. The previous argument that no overall survival has been demonstrated with ART has been invalidated by the recent results from a randomized controlled trial demonstrating that indeed there is overall survival advantage with ART compared with observation and delayed therapy. Thus the main criticisms and concerns regarding ART have been answered and there is convincing and compelling evidence to support the use of ART in patients at high risk of tumour recurrence after radical prostatectomy. Those patients at low risk for tumour recurrence should be vigilantly observed and at the first sign of biochemical or clinical failure, ART should be instituted to optimize treatment response.

Professor and Chair, Division of Surgical Oncology, University of Western Ontario, London, Ont. The positions provided in the Point/Counterpoint series are presented as general information and do not necessarily reflect the personal opinions of the authors.

This article has been peer reviewed.

Competing interests: None declared.

\section{References}

1. Bolla M, van Poppel H, Collette L, et al.; European Organization for Research and Treatment of Cancer. Postoperative radiotherapy after radical prostatectomy: a randomised controlled trial (EORTC trial 22911). Lancet 2005;366:572-4.

2. Thompson IM, Tangen $C M$, Paradelo J, et al. Adjuvant radiotherapy for pathologically advanced prostate cancer: a randomized clinical trial. JAMA 2006;296:2329-35.

3. Chin JL. Post-radical prostatectomy management options for the positive surgical margin: argument for adjuvant radiotherapy. Urol Oncol 2009;27:87-8.

4. Morgan SC, Waldron TS, Eapen L, et al.; Genitourinary Cancer Disease Site Group of the Cancer Care Ontario Program in Evidence-based Care. Adjuvant radiotherapy following radical prostatectomy for pathologic T3 or margin-positive prostate cancer: a systematic review and meta-analysis. Radiother Oncol 2008;88:1-9.

5. Thompson IM, Tangen $C M$, Paradelo J, et al. Adjuvant radiotherapy for pathological T3NOMO prostate cancer significantly reduces risk of metastases and improves survival: long-term follow-up of a randomized clinical trial. J Urol. In press.

6. Swanson GP, Goldman B, Tangen CM, et al.; Southwest Oncology Group 8794. The prognostic impact of seminal vesicle involvement found at prostatectomy and the effects of adjuvant radiation: data from Southwest Oncology Group 8794. J Urol 2008;180:2453-7.

7. Teh BS, Bastasch MD, Mai WY, et al. Long-term benefits of elective radiotherapy after prostatectomy for patients with positive surgical margins. J Urol 2006;175:2097-101.

8. Caraffini B, De Stefani A, Vitali E, et al. Postoperative radiotherapy after radical prostatectomy for prostate carcinoma: the experience of the Brescia Radium Institute. Radiol Med 2006;111:741-7.

9. Pacholke HD, Waisman Z, Algood CB, et al. Postoperative adjuvant and salvage radiotherapy for hemical relapse and survival prostate cancer: impact on freedom from biochemical relapse and survival. Urology 2004;64:982-6.

10. Trabulsi EJ, Valicenti RK, Hanlon AL, et al. A multi-institutional matched-control analysis of adjuvant and salvage postoperative radiation therapy for pT3-4NO prostate cancer. Urology 2008;72:1298-302.

11. Wadasaki K, Kaneyasu Y, Kenjo M, et al. Treatment results of adjuvant radiotherapy and salvage radiotherapy after radical prostatectomy for prostate cancer. Int I Clin Oncol 2007;12:37-41.

12. Loeb S, Roehl KA, Viprakasit DP, et al. Long-term rates of undetectable PSA with initial observation and delayed salvage radiotherapy after radical prostatectomy. Eur Urol 2008;54:88-94.

13. Stephenson AJ, Scardino PT, Kattan MW, et al. Predicting the outcome of salvage radiation therapy for recurrent prostate cancer after radical prostatectomy. J Clin Oncol 2007;25:2035-41.

14. Lennernäs $B$, Edgren $M$, Häggman $M$, et al. Postoperative radiotherapy after prostatectomy - a review. Scand J Neprol Urol 2003;37:10-5.

15. Pasquier D, Ballereau C. Adjuvant and salvage radiotherapy after prostatectomy for prostate cancer: a literature review. Int I Radiat Oncol Biol Phys 2008;72:972-9.

16. Brooks JP, Albert PS, Wilder RB, et al. Long-term salvage radiotherapy outcome after radical prostatectomy and relapse predictors. J Urol 2005; 174:2204-8.

17. Pisansky TM, Kozelsky TF, Myers RP, et al. Radiotherapy for isolated serum prostate specific antigen elevation after prostatectomy. J Urol 2000;163:845-50. 
18. Vees H, Buchegger F, Albrecht S, et al. 18F-choline and/or 11 C-acetate positron emission tomography: detection of residual or progressive subclinical disease at very low prostate-specific antigen valves $(<1 \mathrm{ng} / \mathrm{mL})$ after radical prostatectomy. BJU Int 2007;99:1415-20.

19. Pucar D, Sella $T$, Schöder $H$. The role of imaging in the detection of prostate cancer local recurrence after radiation therapy and surgery. Curr Opin Urol 2008;18:87-97.

20. Bottke D, Wiegel T. pT3RI prostate cancer: Immediate or delayed radiotherapy after radical prostatectomy? [article in German]. Urologe A 2008;47:1431-5.

21. Van Cangh PJ, Ricard F, Lorge F, et al. Adjuvant radiation therapy does not cause urinary incontinence after radical prostatectomy: results of a prospective randomized study. J Urol 1998;159:164-6.

22. Feng MG, Hanlon AL, Pisansky TM, et al. Predictive factors for late genitourinary and gastrointestinal toxicity in patients with prostate cancer treated with adjuvant or salvage radiotherapy. J Urol 2005; 174:2204-8.
23. Pinkawa M, Fischedick K, Asadpour $B$, et al. Health-related quality of life after adjuvant and salvage postoperative radiotherapy for prostate cancer - a prospective analysis. Radiother Oncol 2008;88:135-9.

24. Amos EH, Mendenhall WM, McCarty PJ, et al. Postoperative radiotherapy for locally advanced colon cancer. Ann Surg Oncol 1996;3:431-6.

25. Chen AM, Granchi PJ, Garcia J, et al. Local-regional recurrence after surgery without postoperative irradiation for carcinomas of the major salivary glands: implications for adjuvant therapy. Int I Radiat Oncol Biol Phys 2007;67:982-7.

Correspondence: Dr. Joseph Chin, C3-120C, 800 Commissioners Rd. E., London Health Sciences Centre, London ON N6A 4G5; fax 519 685-8455; joseph.chin@hsc.on.ca

CUAJ is read by at least 3000 urologists and related specialists in Canada and around the world.

If you want to reach urologists, there is no better place for your classified advertisement. Please contact the journal at journal@cua.org or call 514 395-0376 x40 for a price list, specifications and deadlines. 